\title{
La luxation sous-talienne médiale isolée traumatique
}

\section{Traumatic Isolated Medial Subtalar Dislocation}

\section{P. Valero $\cdot$ R. Coulomb}

Reçu le 28 avril 2018 ; accepté le 17 septembre 2018

(C) SFMU et Lavoisier SAS 2018
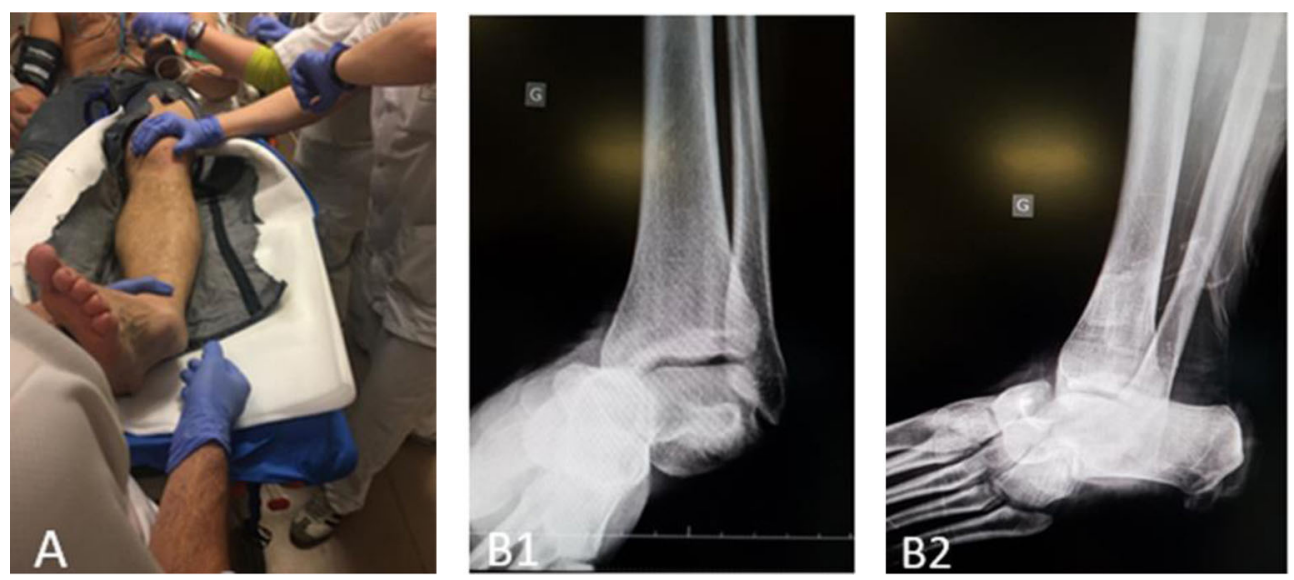

Fig. 1 A. Déformation clinique de la cheville et du pied. B. Bilan radiographique à l'arrivée aux urgences ; incidence de face (B1) et de profil (B2)

La luxation sous-talienne (LST) médiale aiguë pure, sans lésion associée est rare [1-3]. Elle fait suite le plus souvent à un traumatisme en varus équin à haute énergie [1], à une chute de hauteur, à un accident de la route ou à un accident sportif [2,3]. Plus généralement, la LST peut survenir dans n'importe quelle direction, conditionnant les lésions associées dont les plus fréquentes sont une souffrance cutanée et la survenue de fractures (malléolaires, talus ou cinquième métatarsien) [1].

Nous rapportons le cas d'un jeune éleveur, victime d'une LST pure traumatique et pris en charge dans notre centre.

P. Valero $(\bowtie)$

Service des urgences Smur, centre hospitalier Joseph-Imbert,

F-13200 Arles, France

e-mail : philouval@hotmail.com

\section{R. Coulomb}

Service de chirurgie orthopédique et

traumatologique et chirurgie du rachis,

CHU de Nîmes, F-30029 Nîmes cedex 09, France

\section{Cas clinique}

Un patient âgé de 23 ans est admis au service des urgences du centre hospitalier d'Arles, le 25 mars 2018 à 20 h 16 pour un traumatisme de la cheville et du pied gauche suite à un traumatisme direct (coup de sabot sur le pied). Il existe une importante déformation clinique (Fig. 1A).

Le patient est installé en salle de déchocage compte tenu de la présentation clinique (EVA douleur $>7+$ déformation clinique de l'arrière-pied). Dans le même temps sont réalisés le monitorage et la prise en charge de la douleur en utilisant une procédure analgésique associant kétamine $(0,3$ à $0,5 \mathrm{mg} /$ $\mathrm{kg}$ ) et midazolam ( $3 \mathrm{mg}$ pour $70 \mathrm{~kg}$ ) pour permettre une prise en charge rapide des premiers soins. L'examen clinique recherche une souffrance ou une ouverture cutanée en regard de la déformation, la présence des pouls pédieux et tibial postérieur, l'absence de déficit sensitif (plantaire) et moteur (mobilité des orteils) ainsi que la palpation des repères osseux. Cet examen reste difficile en raison de la douleur résiduelle persistante en lien avec la déformation.

Un bilan radiologique de la cheville de face et de profil permet de diagnostiquer une LST, dont le déplacement du 
bloc calcanéopédieux est médial sans lésion ostéoarticulaire associée visible (Fig. 1B1B2). Au retour en salle de déchocage, un traitement orthopédique est décidé en urgence en accord avec notre chirurgien orthopédique de garde. Le médecin urgentiste réduit cette luxation en utilisant du propofol $(0,5$ à $1 \mathrm{mg} / \mathrm{kg})$ par traction douce et rechaussage du bloc calcanéopédieux sous le talus, corrigeant ainsi la déformation et permettant une protection rapide de la peau (facteur pronostique essentiel de ces LST). Un testing clinique confirme la stabilité de l'articulation sous-talienne et l'absence de trouble vasculonerveux. Une attelle plâtrée postérieure et un contrôle radiologique sont effectués après réduction. Un plâtre circulaire a été réalisé à la consultation orthopédique à 72 heures du traumatisme permettant la vérification de l'état cutané et la programmation du scanner de contrôle.

\section{Discussion}

La LST se définit comme une luxation simultanée des articulations talonaviculaire et talocalcanéenne sans fracture majeure du talus [1,2,4-6]. Ainsi, le talus reste en place dans la mortaise tibiofibulaire, et l'articulation calcanéocuboïde est intacte [7]. La LST aiguë représente 1 à $2 \%$ de toutes les luxations articulaires et environ $15 \%$ des lésions péritaliennes $[3,7,8]$. Elles représentent environ $1 \%$ de l'ensemble des lésions traumatiques du pied [4]. Le sex-ratio serait trois à quatre fois plus important chez l'homme [2,3]. Le système actuel de classification reprend des définitions bien connues $[3,9,10]$. Avec $80 \%$ des cas de LST, la forme médiale est la plus commune $[1,6,10]$. Elle est prédominante, car l'articulation sous-talienne présente un grand déplacement en inversion favorisant son instabilité [7]. De plus, il semble que les stabilisateurs passifs latéraux (talocalcanéens et calcanéofibulaires) soient plus faibles que les médiaux (ligaments deltoïdiens et talocalcanéens médiaux) $[2,3,10]$. Une luxation médiale se produit lorsqu'une force d'inversion est appliquée sur le pied en flexion dorsale forcée. Le sustentaculum tali agit alors comme un pivot autour duquel pivote le col du talus, provoquant ainsi la luxation de l'articulation talonaviculaire (rupture du ligament dorsal talonaviculaire) suivie d'une dislocation de l'articulation talocalcanéenne (lésion du ligament talocalcanéen interosseux et calcanéofibulaire). L'os naviculaire coulisse alors médialement par rapport au talus [7]. La LST médiale est généralement le résultat d'un traumatisme à haute énergie (chute d'un lieu élevé, accident de la route, accident sportif) [1-3,7]. Chez certains sportifs, ce mécanisme d'inversion forcée sur un pied déjà inversé est appelé « pied de basket » [1]. Plus rarement ont été décrits des cas de LST pour des accidents à faible cinétique [3].

Ces luxations sont fréquemment associées à des lésions diverses à type d'ouverture cutanée, de fractures (talus : tête, col, processus postérieur du talus ; malléole interne ou externe ; base du cinquième métatarsien) et d'atteintes neurovasculaires $[2,4,10]$. Les radiographies de cheville et du pied de face, de profil et de trois quarts évaluent l'intégrité de l'articulation tibiotalienne, la présence de fractures malléolaires et des os du tarse associées, pouvant modifier l'approche thérapeutique définitive [4]. Pour Lancaster [3], les luxations pures de type I doivent être réduites en urgence, sous procédure analgésique adaptée, afin de minimiser les risques de nécrose cutanée [6,7] en vue du meilleur résultat fonctionnel possible $[3,4,6]$. Plusieurs méthodes de réduction par manœuvres externes ont été décrites [6,7]. La technique communément utilisée nécessite une analgésie ou une anesthésie afin d'obtenir un relâchement musculaire optimum et ainsi réduire les dommages des surfaces articulaires pendant la manipulation. Le genou ipsilatéral est fléchi pour obtenir un relâchement des muscles gastrocnémiens. Une traction longitudinale ferme du pied est appliquée avec une contre-traction sur la jambe combinée initialement à l'accentuation de la déformation. Ce geste est suivi d'un revers de la déformation (éversion pour la luxation médiale et inversion pour la luxation latérale). Une pression directe digitale sur la tête du talus permet d'aider à la réduction qui s'accompagne d'un bruit audible $[1,6]$. Les manœuvres brusques, une force excessive et des tentatives multiples de réduction fermées doivent être évitées afin de ne pas générer des lésions supplémentaires des tissus mous [4]. Une fois la réduction obtenue, l'état neurovasculaire et la stabilité de l'arrière- et du médio-pied doivent être évalués $[4,6]$.

L'immobilisation initiale se fait dans une attelle plâtrée postérieure s'arrêtant sous le genou [4] et pouvant être circularisée secondairement. On s'attache à confirmer radiologiquement que la réduction de cette luxation est adéquate. La période d'immobilisation fréquemment retrouvée dans la littérature est de quatre semaines [2-4,10,12], mais il existe une controverse quant au temps d'immobilisation postréduction [4,13-15]. Dans tous les cas, il semble qu'au-delà de six semaines d'immobilisation la réduction de la mobilité de l'articulation sous-talienne soit excessive selon Heppenstall et al. [16]. La synthèse collagénique nécessaire à la reconstruction ligamentaire est de six semaines. Une protection prenant en compte ce délai semble indispensable, mais pas au-delà, ce qui permet également de prévenir la fibrose de l'articulation sous-talienne et environnementale [14]. On peut conclure que les luxations sous-taliennes traumatiques pures, rapidement réduites et immobilisées quatre semaines, ont une évolution favorable à long terme $[10,12]$. Une botte de marche peut être utilisée les deux semaines suivantes, permettant de commencer la rééducation.

Une tomodensitométrie de cheville postréduction est recommandée pour identifier les fractures occultes ou autres lésions ostéochondrales qui auraient pu passer inaperçues $[2,3,7]$. Ces lésions occultes sont présentes dans 39 à $88 \%$ 
des cas [4]. Dans un deuxième temps, le bilan ligamentaire et des tissus mous pourra être réalisé avec une imagerie par résonance magnétique dont l'intérêt en urgence est nul.

Le devenir de ces LST est émaillé de quatre complications majeures : la raideur est la complication la plus fréquente, l'arthrose, la nécrose avasculaire du talus et l'instabilité de l'articulation sous-talienne $[4,7,10,17]$. Les résultats fonctionnels de ces luxations se réfèrent majoritairement au score spécifique arrière-pied de l'American Orthopaedic Foot and Ankle Society (AOFAS) de 0 à 100.

En conclusion, les LST traumatiques aiguës isolées sont des lésions rares qui surviennent volontiers dans les suites d'un traumatisme à haute énergie. Une prise en charge en urgence s'impose après un bilan clinique, cutané, neurovasculaire et radiologique afin de réaliser en urgence une réduction par manœuvres externes. Les formes pures isolées réduites rapidement dans de bonnes conditions jouissent d'un meilleur résultat clinique et fonctionnel.

Conflits d'intérêts : les auteurs déclarent ne pas avoir de liens d'intérêts.

\section{Références}

1. Gantsos A, Giotis D, Giannoulis DK (2013) Conservative treatment of closed subtalar dislocation: a case report and 2 years follow-up. Foot 23:107-10

2. Rida-Allah B, Aitbenali H, Mahfoud M, et al (2015) Rare case of pure medial subtalar dislocation: conservative treatment and 32 months follow-up. J Emerg Trauma Shock 8:174-5
3. Pesce D, Wethern J, Patel P (2011) Rare case of medical subtalar dislocation from a low-velocity mechanism. Eur J Emerg Med 6:121-4

4. Prada-Canizares A, Aunon-Martin I, Rico JV, et al (2016) Subtalar dislocation: management and prognosis for an uncommon orthopaedic condition. Int Orthop 40:999-1007

5. Gaba S, Kumar A, Triha V, Das S, et al (2017) Luxation postérieure de l'articulation sous-astragalienne sans fracture associée : rapport de cas et revue de la littérature. J Clin Diagn Res 11:1-2

6. Ruhlmann F, Poujardieu C, Vernois J, et al (2017) Isolated acute traumatic subtalar dislocations: review of 13 cases at a mean follow-up of 6 years and literature review. J Foot Ankle Surg 56:201-7

7. DeLee JC, Curtis R (1982) Subtalar of the foot. J Bone Joint Surg Am 64:433-7

8. Krishnan KM, Sinha AK (2003) La vraie luxation postérieure de l'articulation sous-astragalienne : un cas. J Foot Ankle Surg 42:363-5

9. Pérouse D, Basile A, Terrence J, et al (2007) Dislocation soustalienne antéromédiale. J Foot Ankle Surg 46:52-4

10. Inokuchi S, Hashimoto T, Usami N, et al (1996) Subtalar dislocation of the foot. Foot 6:168-74

11. Perugia D, Basile A, Massoni C, et al (2002) Conservative treatment of subtalar dislocation. Int Orthop 26:56-60

12. Giannoulis D, Papadopoulos VD, Lykissas GM, et al (2015) Luxation sous-talienne sans fracture associée : rapport de cas et revue de la littérature. WJO 6:374-9

13. McKeever FM (1963) Treatment of complications of fractures an dislocations of the talus. Clin Orthop Relat Res 30:45-52

14. Buckingham WW Jr, LeFlore I (1973) Subtalar dislocation of the foot. J Trauma 13:753-765

15. De Palma L, Santucci A, Marinelli M, et al (2008) Clinical outcome of closed subtalar dislocations. Arch Ortho Trauma Surg 128:593-8

16. Heppenstall RB, Farahvar H, Balderston R, et al (1979) Subtalar dislocation with medial or lateral displacement. Rev Chir Ortho Reparatrice Appar Mot 65:377-85

17. Nkaoui M, Boufettal M, Sasbou Y, et al (2017) Luxation soustalienne pure : à propos d'un cas. PAMJ 27:123 